\title{
Conditional survival analysis in Korean patients with gastric cancer undergoing curative gastrectomy
}

\author{
Jin Won Lee, Bandar Ali, Han Mo Yoo, Cho Hyun Park and Kyo Young Song*
}

\begin{abstract}
Background: Conditional survival (CS) measures the probability that patients will survive an additional number of years given that they have already survived for a certain period of time.

Methods: In total, 2935 gastric cancer patients who had undergone curative gastrectomy between 1995 and 2011 were enrolled. The Cox proportional hazard regression model was used to evaluate the factors associated with overall survival (OS). Three-year CS estimates at ' $t$ ' years after surgery were calculated as follows: $C S(t)=S(t+3) / S(t)$.

Results: The 1-, 2-, 3-, 4- and 5-year OS rates of the 2935 patients were $96.6 \%, 92.0 \%, 88.7 \%, 85.6$ and $82.7 \%$, respectively. The probability of surviving an additional 3 years on the condition of having already survived 1, 2, 3, 4 and 5 years after surgery were $88.6 \%, 89.9 \%, 91.0 \%, 92.2 \%$ and $93.2 \%$, respectively. Patients with a higher risk at baseline showed a greater increase in CS over time.

Conclusions: CS estimates provide important information about dynamic prognostic changes over time for Korean gastric cancer patients, and as such, can be used to guide long-term follow-up strategies.
\end{abstract}

Keywords: Survival, Gastric cancer, Gastrectomy, Prognosis, Follow-up

\section{Background}

Despite declining global incidence rates, gastric cancer remains the fourth most common malignancy and the second leading cause of worldwide cancer-related mortality [1]. The cornerstone of curative therapy for gastric cancer remains surgical resection with adequate lymphadenectomy. In recent years, there have been many advances in treatment options, including the establishment of surgical techniques for tumor resection and lymph node dissection. Together with progress in adjuvant chemotherapy, radiotherapy, and molecular-targeted therapy, the long-term outcome of patients with gastric cancer has improved considerably. Therefore, to maximize the efficacy of treatment and follow-up strategy, it is important to establish the prognoses of individual patients and to apply suitable treatment strategies.

\footnotetext{
* Correspondence: skys9615@gmail.com

Division of Gastrointestinal Surgery, Department of Surgery, Seoul St. Mary's Hospital, College of Medicine, The Catholic University of Korea, 222 Banpo-daero, Seocho-gu, Seoul 137-701, South Korea
}

Previous comparisons of risk-grouping approaches in several diseases using nomograms have shown improved predictive accuracies. A further potential benefit of nomograms is that, through their simple graphical representation of a statistical predictive model, they generate a numerical probability of a clinical event [2].

In general, cancer patient survival is calculated from the day of operation to the most recent follow-up visit or death. Traditional survival estimation and initial prognosis assessment at the time of surgery facilitate adjuvant chemotherapy selection and follow-up scheduling. This approach, however, lacking postoperative follow-up information, provides only a static view of risk, because the prognosis of patients who have already survived for a certain period of time after their initial treatment is not the same as the initial prognosis [3, 4]. Furthermore, patients often ask questions regarding their probability of survival from the time they begin to visit a clinic for follow-ups, but physicians and clinicians are unable to adequately respond based only on the 5-year overall survival (OS) reported at the time of surgery. 
Conditional survival (CS) estimates are based on the concept of conditional survival probability [5]. They represent the probability of surviving additional years conditional to having already survived for a certain period. Thus they account for changes in hazard rate over time as well as for dynamic changes in prognosis. The usefulness of CS estimates has been established for many solid malignancies including urothelial, colorectal, thyroid, ovary, breast, lung and gastric tumors [3, 4, 6-15]. A multicenter analysis by the US Gastric Cancer Collaborative indicated that CS estimates provided important information on changes in the probability of survival over time [16].

However, to date there has been no comparable study on Korean patients with gastric cancer. The aims of this study, therefore, were to estimate, based on the analysis of a large-scale database, the CS of Korean gastric cancer patients and to determine its usefulness to those patients' prognoses.

\section{Methods}

\section{Patients and data collection}

In total, 2935 patients who had undergone curative radical gastrectomy at our hospital between January 1995 and December 2011 were included in this study. The relevant demographic, preoperative, postoperative, and pathologic data were collected from the patients' medical records. Preoperative disease assessment was based on physical examinations, blood tests, chest and abdominal X-rays, endoscopy, abdominal computed tomography scans and positron emission scanning. Pathological staging was assigned using The American Joint Committee on Cancer (AJCC; $7^{\text {th }}$ ed.) [17], in which depth of invasion is defined by $\mathrm{T} 1$ tumor invasion of the mucosa and submucosa, T2 tumor invasion of the muscularis propria, T3 tumor penetration of the subserosal connective tissue without invasion of the visceral peritoneum, and T4 tumor invasion of the serosa (T4a) or adjacent organs (T4b). Data on recurrence and overall survival (OS) were collected. Approval for this study was obtained from the Institutional Review Board of Seoul St. Mary's Hospital (KC15RI0252). The research was conducted in compliance with the Helsinki Declaration.

Only patients undergoing resection with curative intent were enrolled. Patients undergoing palliative resection and those with known metastatic disease, 30-day perioperative mortalities, and a history of other organ malignancies were excluded. For each patient, the following parameters were recorded: age, gender, performance status according to the Eastern Cooperative Oncology Group (ECOG) scale, tumor location, resection extent (total gastrectomy, subtotal gastrectomy and others), main-tumor size $(\mathrm{cm})$, histologic type (differentiated type: well or moderately differentiated tubular and papillary adenocarcinoma; undifferentiated type: poorly differentiated adenocarcinoma, signet-ring cell or mucinous carcinoma), lymphatic invasion (LI), vascular invasion (VI), neural invasion (NI), Lauren classification (intestinal, diffuse or mixed type), tumor-node metastasis (TNM) stage, number of retrieved lymph nodes, and number of positive nodes.

\section{Conditional survival (CS) estimates}

The primary outcome was 3-year $\mathrm{CS}\left(\mathrm{CS}_{3}\right)$ at each time point. CS is the estimation of the probability that a patient will survive for an additional number of years given that he or she has already survived a certain period of time [17]. The $\mathrm{CS}_{3}$ at ' $\mathrm{t}$ ' years after surgery is defined as the probability of surviving an additional 3 years after $t$ years. This was calculated as $\mathrm{CS}(\mathrm{t})(\%)=[\mathrm{S}(\mathrm{t}+3) / \mathrm{S}(\mathrm{t})] \times$ 100. The Kaplan-Meier log-rank test and the Cox proportional hazard regression model were used to evaluate the factors associated with OS. CS was compared with OS according to the variables determined by multivariate analysis to be independent risk factors for survival.

\section{Statistical analysis}

Continuous data were expressed as means \pm standard deviation. The independent factors significantly related to patient survival were evaluated with reference to statistics obtained by the Kaplan-Meier method for calculating OS, and the multivariate Cox proportional hazard model was utilized to assess the effects of the variables on OS. All of the tests were two-sided; statistical significance was predefined at $P<0.05$. All of the statistical analyses were performed using SPSS software (version 12.0; SPSS, Chicago, IL, USA).

\section{Results}

\section{Patient characteristics}

The baseline characteristics of the 2935 patients are shown in Table 1 . The mean age was 57.8 years, and $66 \%(n=$ 1424) were male. The majority of patients had a performance status of 0 or 1 . At the time of surgery, the great majority of patients underwent either a subtotal gastrectomy $(75.4 \%)$ or a total gastrectomy (24.0\%), with the remaining $0.6 \%$ undergoing other operations (either Whipple's procedure, proximal gastrectomy or wedge resection). The mean tumor size was $4.07 \mathrm{~cm}$. With regard to the Lauren classification, $43.7 \%$ of patients had an intestinal-type tumor, while the remaining tumors were either diffuse or of the mixed type. Most tumors were located in the lower third of the stomach $(n=1632,55.6 \%)$, and half of the tumors were early-stage cancer (T1 tumors: $n=1594,54.5$ $\%)$. Based on the The AJCC ( $7^{\text {th }}$ ed.) staging system, most of the patients had stage I tumors $(n=1743,59.4 \%)$; the remaining patients had either stage II $(n=553,18.8 \%)$ or stage III $(n=639,21.8 \%)$ tumors (Table 1$)$. 
Table 1 Clinicopathologic characteristics associated with survival in 2935 patients after curative gastrectomy for gastric cancer

\begin{tabular}{|c|c|c|c|}
\hline & No (\%) & 5-YSR & $P^{*}$ value \\
\hline Age & & & 0.000 \\
\hline
\end{tabular}

Mean \pm SD

$<60$

$\geq 60$

Sex

Male

Female

ECOG PS

0

1

2

3

4

5

Unknown

Resection extent

TG

STG

Others

Tumor size

Mean \pm SD

$<5 \mathrm{~cm}$

$\geq 5 \mathrm{~cm}$

LI

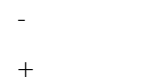

Unknown

VI

$\begin{array}{ll}- & 2762(94.1) \\ + & 141(4.8) \\ \text { Unknown } & 26(0.9)\end{array}$

NI

$\begin{array}{ll}- & 2206(75.2) \\ + & 697(23.7) \\ \text { Unknown } & 25(0.9)\end{array}$

Leuran classification

Intestinal

Diffuse

Mixed

Unknown

Histology

Differentiated

Undifferentiated
1283 (43.7)

$57.8 \pm 11.95$

1511 (51.5)

86.2

78.8

$1424(48.5)$

1931 (65.8)

82.0

83.9

1272 (43.3)

90.6

78.2

53.5

50.0

0

-

85.0

73.3

85.7

68.1

$19(0.6)$

$4.07 \pm 2.80$

2004 (68.3)

931 (31.7)

1784 (60.8)

1130 (38.5)

$16(0.5)$

$25(0.9)$

993 (33.8)

601 (20.5)

$58(2.0)$

$1358(46.3)$

$1577(53.7)$

89.4

62.2
Table 1 Clinicopathologic characteristics associated with survival in 2935 patients after curative gastrectomy for gastric cancer (Continued)

\begin{tabular}{|c|c|c|c|}
\hline Location & & & 0.000 \\
\hline Esophagus & $34(1.3)$ & 72.3 & \\
\hline Upper 1/3 & $286(9.7)$ & 80.6 & \\
\hline Middle 1/3 & 947 (32.3) & 83.2 & \\
\hline Lower 3 & $1632(55.6)$ & 82.9 & \\
\hline Duodenum & $1(0)$ & 100 & \\
\hline Whole Stomach & $11(0.4)$ & 53.3 & \\
\hline Unknown & $24(0.8)$ & 100 & \\
\hline Depth & & & 0.000 \\
\hline $\mathrm{T} 1$ & $1596(54.4)$ & 96.0 & \\
\hline $\mathrm{T} 2$ & $346(11.8)$ & 87.4 & \\
\hline T3 & $532(18.1)$ & 71.6 & \\
\hline T4a & $442(15.1)$ & 47.7 & \\
\hline T4b & $17(0.5)$ & 48.7 & \\
\hline \multicolumn{4}{|l|}{ Total number of } \\
\hline \multicolumn{4}{|l|}{ Retrieved LN } \\
\hline Mean \pm SD & $41.62 \pm 16.27$ & & \\
\hline \multicolumn{4}{|l|}{ Total number of } \\
\hline \multicolumn{4}{|l|}{ Positive LN } \\
\hline Mean \pm SD & $2.56 \pm 5.95$ & & \\
\hline Nodal status & & & 0.000 \\
\hline NO & $1887(64.3)$ & 94.3 & \\
\hline $\mathrm{N} 1$ & $366(12.5)$ & 79.8 & \\
\hline N2 & $308(10.5)$ & 71.2 & \\
\hline N3 & $373(12.7)$ & 39.3 & \\
\hline TNM Stage & & & 0.000 \\
\hline I & 1743 (59.4) & 96.1 & \\
\hline$\|$ & $553(18.8)$ & 80.9 & \\
\hline III & 639 (21.8) & 49.7 & \\
\hline
\end{tabular}

Continuous variables are presented as the mean \pm standard deviation No, number of patients; 5-YSR, 5-year survival rate; ECOG Eastern cooperative oncology group, PS Performance status, TG Total gastrectomy, STG Subtotal gastrectomy, LI Lymphatic invasion, VI Venous invasion, NI Neural invasion, LN Lymph node, TNM Tumor-node-metastasis

0.000 Log-rank test

Prognostic factors associated with overall survival (OS)

0.000 Baseline demographic and tumor-characteristic variables associated with survival were selected for analysis. A univariate analysis using the Kaplan-Meier method identified all of the factors, except gender, as having a significant association with outcome. According to the multivariate analysis, age, performance status, tumor size, tumor depth, nodal status, and TNM stage were each independently associated with OS (all $P<0.05$ )(Table 2), which results are consistent with the findings of relevant previous studies $[16,18]$. 
Table 2 Independent prognostic factors associated with time to death at multivariate analysis using cox proportional hazards model

\begin{tabular}{|c|c|c|c|}
\hline & $\mathrm{HR}$ & $95 \% \mathrm{Cl}$ & $P$ value \\
\hline Age & & & 0.000 \\
\hline$<60$ & Ref & - & \\
\hline$\geq 60$ & 1.760 & $1.489-2.079$ & \\
\hline ECOG PS & & & 0.001 \\
\hline $0-1$ & Ref & - & \\
\hline $2-5$ & 1.968 & $1.317-2.940$ & \\
\hline Tumor size & & & 0.014 \\
\hline$<5 \mathrm{~cm}$ & Ref & & \\
\hline$\geq 5 \mathrm{~cm}$ & 1.267 & $1.050-1.529$ & \\
\hline Depth & & & 0.000 \\
\hline $\mathrm{T} 1$ & Ref & - & \\
\hline $\mathrm{T} 2$ & 1.499 & $1.097-2.050$ & \\
\hline $\mathrm{T} 3$ & 2.160 & $1.640-2.845$ & \\
\hline T4a & 3.153 & $2.338-4.253$ & \\
\hline $\mathrm{T} 4 \mathrm{~b}$ & 4.910 & $2.504-9.628$ & \\
\hline Nodal status & & & 0.000 \\
\hline No & Ref & - & \\
\hline N1 & 1.151 & $0.820-1.616$ & \\
\hline N2 & 1.379 & $0.979-1.942$ & \\
\hline N3 & 2.863 & $2.062-3.976$ & \\
\hline TNM Stage & & & 0.000 \\
\hline I & Ref & - & \\
\hline$\|$ & 2.328 & $1.752-3.093$ & \\
\hline III & 3.387 & $2.235-5.133$ & \\
\hline
\end{tabular}

$H R$ Hazard ratio, $\mathrm{Cl}$ Confidence interval, ECOG Eastern cooperative oncology group, PS Performance status, TNM Tumor-node-metastasis

\section{Overall (OS) and conditional survival (CS) estimates}

1) Overall patients

Figure 1 shows that the OS decreased over time, whereas the $\mathrm{CS}_{3}$ increased. The OS at 3 years, 88.7 $\%$, decreased to $80.7 \%$ at 6 years. By contrast, the $\mathrm{CS}_{3}$ at 3 years, indicating the probability of surviving an additional 3 years postoperatively, was $91 \%$.

2) High-risk patients

The calculated $\mathrm{CS}_{3}$ for the strata of the prognostic factors found on multivariate analysis exceeded the OS, and the gaps between the OS and CS increased more prominently in patients with higher risk factors (i.e., those initially predicted to have a poor outcome) (Fig. 2). For example, the $\mathrm{CS}_{3}$ at 1 - and 4-year $\mathrm{OS}$ in patients with a tumor size of $5 \mathrm{~cm}$ or more were 75.7 and $70.6 \%$, respectively. This difference between $\mathrm{CS}_{3}$ and OS increased, over time, to $36.7 \%\left(\mathrm{CS}_{3}\right.$ at 7 years: $92.8 \%$; 10-year OS: $56.1 \%$ ) (Fig. 2a). Similarly, the
$\mathrm{CS}_{3}$ at 7 years in patients diagnosed with N2 and N3 were 92.8 and $92.5 \%$, respectively, whereas the 10 -year OS were 60.7 and $31.0 \%$, respectively (Fig. 2b). This trend was prominent also for the $\mathrm{T}$ stage (depth of invasion) and overall stage. For example, the $\mathrm{CS}_{3}$ of patients with T4b disease increased from 56.3 to 100 $\%$. Finally, the $\mathrm{CS}_{3}$ in patients with stage III disease increased from 64.7 to $95.1 \%$, respectively (Fig. 2d). Interestingly, for patients who were at a more advanced disease stage, the $\mathrm{CS}_{3}$ was higher at the end of the study period.

3) Low-risk patients

The $\mathrm{CS}_{3}$ at 1- and 4-year OS in patients with a tumor size less than $5 \mathrm{~cm}$ were 94.5 and $92.7 \%$, respectively, and the difference between $\mathrm{CS}_{3}$ and OS after 6 additional years increased only to $11.5 \%\left(\mathrm{CS}_{3}\right.$ at 7 years: 94.1; 10 year OS: 82.6 \%) (Fig. 2a) Furthermore, the $\mathrm{CS}_{3}$ at 7 years in patients diagnosed with N0 and N1 were 94.0 and $93.4 \%$, respectively, whereas the 10-year OS were 85.7 and $72.8 \%$, respectively (Fig. 2b). Lastly, the $\mathrm{CS}_{3}$ of patients with T1 disease decreased slightly from 97.6 to $94.9 \%$ (Fig. 2c), and that of patients with stage I disease decreased similarly, from 97.7 to $94.5 \%$ (Fig. 2d).

\section{Discussion}

The prognosis of gastric cancer patients usually is evaluated in light of the postoperative pathological findings at the time of initial treatment [18]. In fact, the initial stage of gastric cancer is defined by the AJCC as one of its most important prognostic indices, based on tumor-associated factors such as extent of primary tumor $(\mathrm{T})$, regional lymph nodes $(\mathrm{N})$, and distant metastases $(\mathrm{M})$ at the time of surgery, thus providing staged grouping based on $\mathrm{T}, \mathrm{N}$ and $\mathrm{M}$ [17]. Thus far, many factors have been shown to be of prognostic significance to the 5-year survival rate. These include TNM stage, grade, primary tumor size, tumor location, lymphatic and vascular invasion, age, and gender [19]. As shown by multivariate analysis, age, performance status, TNM stage and tumor size are independent prognostic factors for survival. However, the prognosis of patients at the same stage is not completely homogeneous, and the risk-factor hazard function is not constant over time. Although prognostic predictions are useful for guiding the selection of the treatment modality, they might lose their accuracy once a patient passes the predicted milestone.

Our present results provide information pertinent to the conditional 3-year survival rates of patients with gastric cancer. In this study, as the postoperative time progressed, the next 3-year survival rates for patients in each group, stratified by various factors, not only improved with prolonged survival, but also became similar 

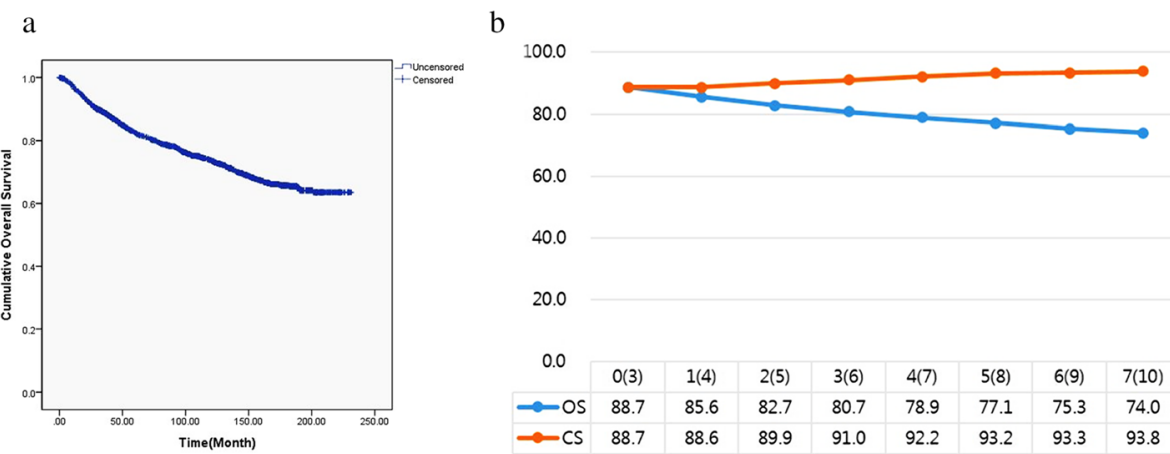

Fig. 1 Survival analysis. a Overall survival (OS) curve of 2935 enrolled patients, b 3-year conditional survival (CS) relative to actuarial survival. The number in parentheses represents the exact OS time-point after surgery

in value by the end of the study period. The OS rates decreased with time, whereas the $\mathrm{CS}_{3}$ estimates increased with time. Yoshihiro et al. [18] concluded that survival is not only stage dependent at the time of surgery but also dependent on the length of survival. They emphasized that prognoses at 1, 2, 3, 4 and 5 years after the initial operation differed from those estimated just after surgery, due to the uncertainty and heterogeneity of the characteristics of this disease at the time of surgery. Correspondingly, our findings suggest that the probability of patient survival evolves over time, and that the impacts of initial prognostic factors decrease with increasing survival $[16,18]$. We found that CS increases markedly over time, especially in patients with late-stage disease.

The CS estimate, a novel prognostic index, provides new information relevant to the dynamic prognostic changes that occur for patients with gastric cancer. Several studies have shown that CS estimates are highly significant to the evaluation of the prognostic prospects of patients with various malignant diseases $[4,6-16,20]$. Furthermore, it is generally accepted that patients with poor prognostic features at the time of initial diagnosis show, compared with those without these features, greater increases in CS over time. Kim et al. [16] demonstrated that patients at higher risk at baseline showed the greatest increases in CS over time. In their study analyzing 807 patients who had undergone resection for gastric adenocarcinoma, the 1-, 3- and 5-year OS rates after gastric resection were 42,34 , and $30 \%$, respectively. The CS estimates, which is to say, the probabilities of surviving an additional 3 years given that the patient had already survived 1,3 , and 5 years, were 56, 71, and $82 \%$, respectively. Our present 1-, 3- and 5-year OS rates after initial surgery were much higher than Kim et al.s: 96.6, 88.7 and $82.7 \%$, respectively. The discrepancies between the two cohorts probably are attributable to the fact that our study population included a relatively high proportion of patients diagnosed with early-stage disease at presentation. The CS estimates of our patients' probabilities of surviving an additional 3 years given their initial 1-, 3- and 5-year survivals were 88.6, 91.0 and $93.2 \%$, respectively. The subsequent 3-year survival rates of patients with worse prognostic factors appeared to approximate, as time passed, those of patients without such risk factors, and the patients with poorer prognostic factors (i.e., tumor size $\geq 5 \mathrm{~cm}$, T4b, $\mathrm{N} 3$, stage III) showed the greatest increases in CS over time. This is due to the fact that the death hazard in patients with gastric cancer is highest during the first few years after the initial surgery, and those who are expected to die based on the estimated 5-year OS typically experience disease recurrence and death during that period [8].

There is a critical limitation to this study. Although we enrolled relatively large numbers of patients, selection bias might have been occurred in diagnosis, treatment, and the follow-up schedule, due simply to the study's retrospective nature. Also, comorbidity data, adjuvant chemotherapy treatment, socioeconomic status and educational level were not evaluated as factors in predicting survival.

In recent years, patients with gastric cancer have been able to survive longer than those diagnosed a few decades ago [1]. This is thanks partly to higher rates of early detection, the development of better chemotherapeutic agents, and improved postoperative care. More than $50 \%$ of patients undergoing gastrectomy in Korea are diagnosed with early gastric cancer. This positive trend, however, has necessitated more accurate prognostic assessment for correspondingly enhanced treatment planning and follow-up strategies [8, 21]. Since many Korean gastric cancer patients have a good chance for long-term survival, CS estimation is even more important than for Western patient populations. 
a

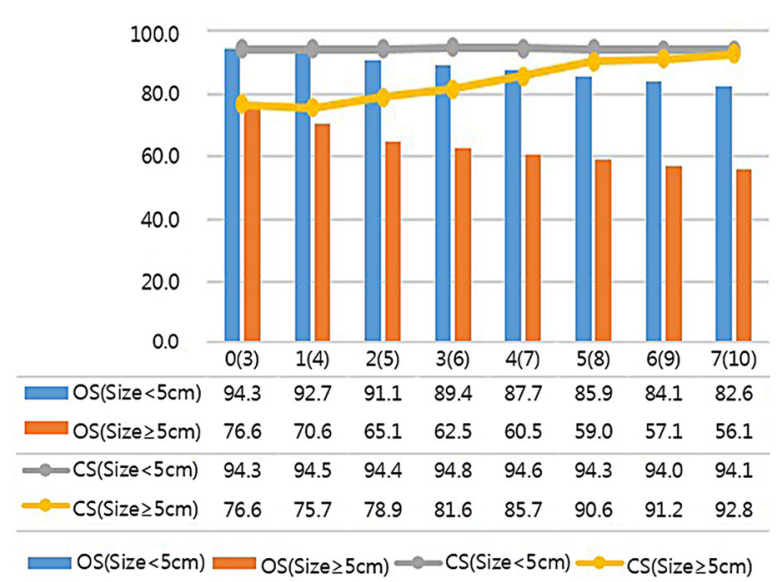

C

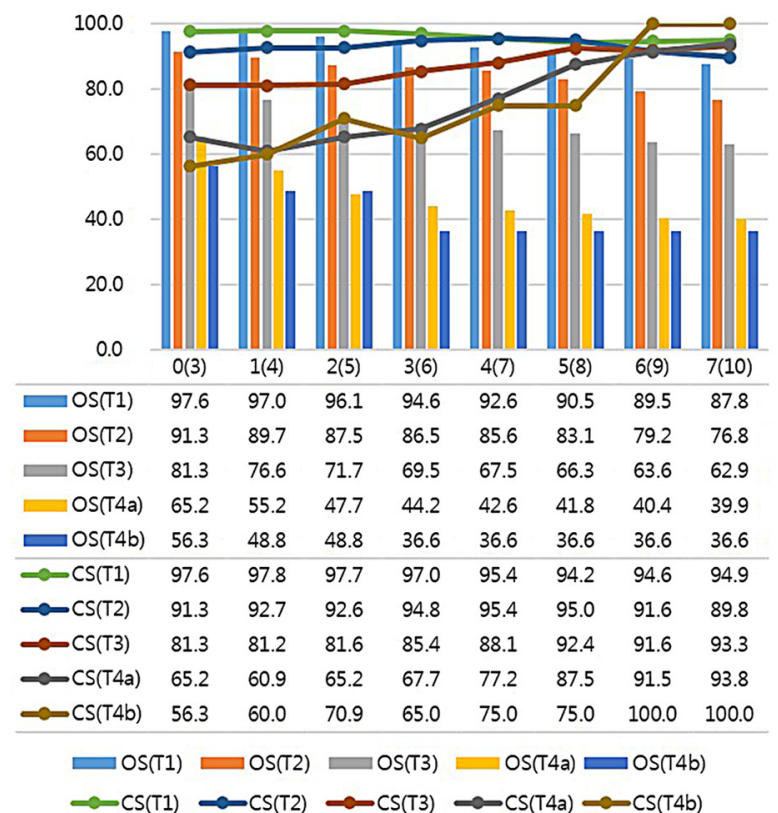

b

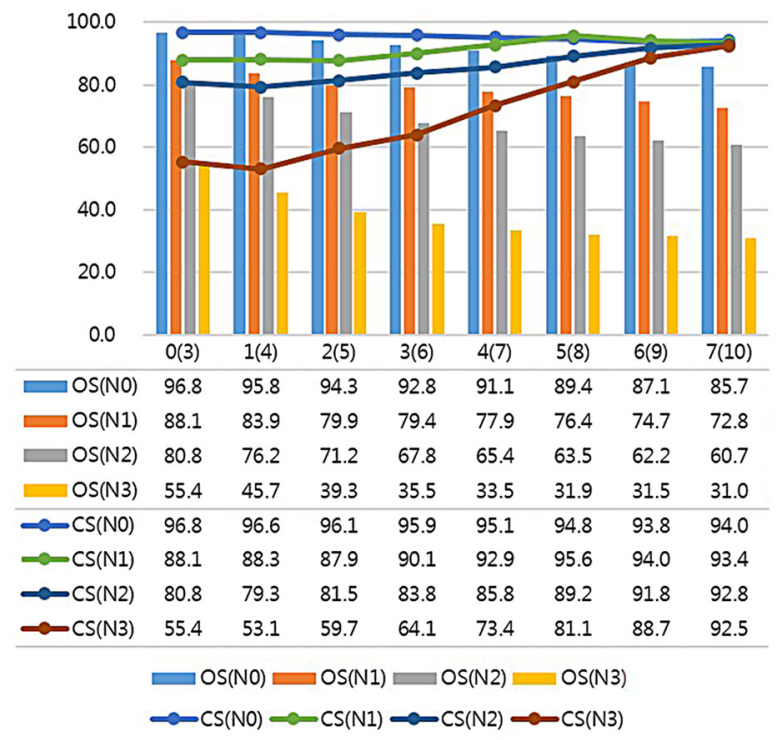

d

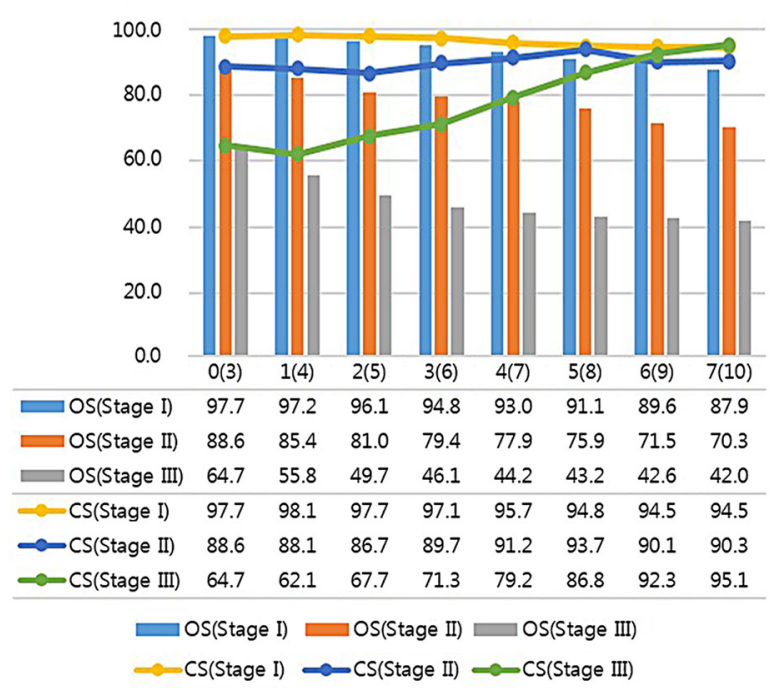

Fig. 2 Comparison of OS with CS, stratified by a tumor size, $\mathbf{b}$ nodal status, $\mathbf{c}$ tumor depth, and $\mathbf{d}$ AJCC stage. The number in parentheses represents the exact OS time-point after surgery

\section{Conclusions}

In conclusion, we demonstrated that CS improves over time following resection for gastric cancer. The adoption of CS estimates can help surgeons and oncologists to better predict survival, make the most appropriate treatment decisions, and conduct a more fully informed discussion with patients in light of their survival expectancy or prognosis. This is very important, particularly as many patients are followed closely for years after surgery, and naturally, they desire to know how long they can expect to survive. For further work, the risk-factor hazard function, the impact of which on survival varies over time, should be evaluated. Moreover, detailed knowledge of time-dependent risk profiles is required for improved prediction of survival and better-individualized followup strategies. 


\section{Consent}

Approval for this study was obtained from the Institutional Review Board of Seoul St. Mary's Hospital Written informed consent from the patient for the publication was waived because this report is retrospective study.

\section{Abbreviations}

CS: Conditional survival; TNM: Tumor-node metastasis; AJCC: American joint committee on cancer; ECOG: Eastern cooperative oncology group;

OS: Overall survival; LI: Lymphatic invasion; Vl: Venous invasion; Nl: Neural invasion.

\section{Competing interests}

The authors declare that they have no competing interests.

\section{Authors' contributions}

JW contributed to the conception and design, carried out the data acquisition and analysis, and drafted the manuscript. BA carried out the data acquisition and interpretation, drafted the manuscript and revised it. HM assisted the study design and performed the statistical analysis. CH conceived of the study, participated in its design and coordination and helped to draft the manuscript. $\mathrm{KY}$ contributed to the conception and design, analyzed and interpreted the data, and revised the manuscript. All of the authors read and approved the final manuscript.

\section{Acknowledgment}

We thank Hyun Kyo Kim of the Department of Surgery, Seoul St. Mary's Hospital of Catholic University, for the substantial data-acquisition contribution.

\section{Funding}

There was no funding for this study.

Received: 11 September 2015 Accepted: 15 December 2015 Published online: 23 December 2015

\section{References}

1. Strong VE, Song KY, Park CH, Jacks LM, Gonen M, Shah M, et al. Comparison of gastric cancer survival following Ro resection in the United States and Korea using an internationally validated nomogram. Ann Surg. 2010;251: 640-6.

2. Song $\mathrm{KY}$, Park YG, Jeon $\mathrm{HM}$, Park $\mathrm{CH}$. A nomogram for predicting individual survival of patients with gastric cancer who underwent radical surgery with extended lymph node dissection. Gastric Cancer Off J Int Gastric Cancer Assoc Jpn Gastric Cancer Assoc. 2014:17:287-93.

3. Ploussard G, Xylinas E, Lotan Y, Novara G, Margulis V, Rouprêt M, et al. Conditional survival after radical nephroureterectomy for upper tract carcinoma. Eur Urol. 2015;67:803-12.

4. Harshman LC, Xie W, Bjarnason GA, Knox J, MacKenzie M, Wood L, et al. Conditional survival of patients with metastatic renal-cell carcinoma treated with VEGF-targeted therapy: a population-based study. Lancet Oncol. 2012; 13:927-35.

5. Henson DE, Ries LA. On the estimation of survival. Semin Surg Oncol. 1994; 10:2-6.

6. Bischof DA, Kim Y, Dodson R, Jimenez MC, Behman R, Cocieru A, et al. Conditional disease-free survival after surgical resection of gastrointestinal stromal tumors: a multi-institutional analysis of 502 patients. JAMA Surg. 2015;150:229-306

7. Youlden DR, Baade PD, Hallahan AR, Valery PC, Green AC, Aitken JF. Conditional survival estimates for childhood cancer in Australia, 2002-2011: a population-based study. Cancer Epidemiol. 2015;150:229-306.

8. Ito Y, Nakayama T, Miyashiro I, loka A, Tsukuma H. Conditional survival for longer-term survivors from 2000-2004 using population-based cancer registry data in Osaka, Japan. BMC Cancer. 2013;13:304.

9. van Erning FN, van Steenbergen LN, Lemmens VEPP, Rutten HJT, Martijn H, van Spronsen DJ, et al. Conditional survival for long-term colorectal cancer survivors in the Netherlands: who do best? Eur J Cancer. 2014;50:1731-9.

10. Banerjee M, Muenz DG, Worden FP, Wong SL, Haymart MR. Conditional survival in patients with thyroid cancer. Thyroid. 2014;24:1784-9.

11. Santoni M, Crabb SJ, Conti A, Rossi L, Burattini L, Conteduca V, et al. Conditional Survival of Patients Treated With First-Line Chemotherapy for Metastatic Urothelial Cancer. Clin Genitourin Cancer. 2014;13:244-9.
12. Zabor EC, Gonen M, Chapman PB, Panageas KS. Dynamic prognostication using conditional survival estimates: conditional survival estimates. Cancer. 2013;119:3589-92

13. Ito Y, Miyashiro I, Ito H, Hosono S, Chihara D, Nakata-Yamada K, et al. Long-term survival and conditional survival of cancer patients in Japan using population-based cancer registry data. Cancer Sci. 2014;105:1480-6.

14. Kurta ML, Edwards RP, Moysich KB, McDonough K, Bertolet M, Weissfeld JL, et al. Prognosis and conditional disease-free survival among patients with ovarian cancer. J Clin Oncol. 2014;32:4102-12

15. Tan MCB, Butte JM, Gonen M, Kemeny N, Fong Y, Allen PJ, et al. Prognostic significance of early recurrence: a conditional survival analysis in patients with resected colorectal liver metastasis. HPB. 2013;15:803-13.

16. Kim Y, Ejaz A, Spolverato G, Squires MH, Poultsides G, Fields RC, et al. Conditional survival after surgical resection of gastric cancer: a multi-institutional analysis of the US gastric cancer collaborative. Ann Surg Oncol. 2015;22:557-64.

17. Edge SB, Compton CC. The American joint committee on cancer: the 7th edition of the AJCC cancer staging manual and the future of TNM. Ann Surg Oncol. 2010;17:1471-4.

18. Moriwaki Y, Kunisaki C, Kobayashi S, Harada H, Imai S, Kido Y, et al. Progressive improvement of prognosis for patients with gastric cancer (dynamic stage grouping) with increasing survival interval from initial staging: how much longer can a given survivor expect to live? Surgery. 2003;133:135-40.

19. Dicken BJ, Bigam DL, Cass C, Mackey JR, Joy AA, Hamilton SM. Gastric adenocarcinoma: review and considerations for future directions. Ann Surg. 2005;241:27-39.

20. Luyimbazi D, Nelson RA, Choi AH, Li L, Chao J, Sun V, et al. Estimates of conditional survival in gastric cancer reveal a reduction of racial disparities with long-term follow-up. J Gastrointest Surg. 2015;19:251-7.

21. Hartgrink HH, Jansen EPM, van Grieken NCT, van de Velde CJH. Gastric cancer. Lancet. 2009:374:477-90.
Submit your next manuscript to BioMed Central and we will help you at every step:

- We accept pre-submission inquiries

- Our selector tool helps you to find the most relevant journal

- We provide round the clock customer support

- Convenient online submission

- Thorough peer review

- Inclusion in PubMed and all major indexing services

- Maximum visibility for your research

Submit your manuscript at www.biomedcentral.com/submit
Biomed Central 